\title{
Upregulation of Wnt signaling under hypoxia promotes lung cancer progression
}

\author{
CHUN-FU HONG ${ }^{1}$, WEI-YOU CHEN $^{2}$ and CHENG-WEN WU ${ }^{2-5}$ \\ ${ }^{1}$ Department of Long-Term Care, National Quemoy University, Kinmen County 89250; \\ ${ }^{2}$ Institute of Biochemistry and Molecular Biology, ${ }^{3}$ Institute of Clinical Medicine, \\ ${ }^{4}$ Institute of Microbiology and Immunology, National Yang Ming University, Taipei 11221; \\ ${ }^{5}$ Institute of Biomedical Science, Academia Sinica, Taipei 11221, Taiwan, R.O.C.
}

Received February 9, 2017; Accepted June 19, 2017

DOI: $10.3892 /$ or.2017.5807

\begin{abstract}
The hypoxic tumor microenvironment induces epithelial-mesenchymal transition (EMT) in tumor cells and increases tumor cell malignancy. Previous studies indicated that malfunction of Wnt signaling is observed in some lung cancer patients. Athough crosstalk between hypoxia and Wnt signaling in tumor cells has recently been revealed, the detailed underlying mechanisms have not been well defined. In the present study, we demonstrated that hypoxia in lung adenocarcinoma cells can enhance Wnt signaling activity by stabilizing $\beta$-catenin and altering its localization into the nucleus. Overexpression of HIF- $2 \alpha$ increased $\beta$-catenin expression, promoted cell mobility, and induced morphological changes to a greater degree than HIF-1 $\alpha$ overexpression. Knockdown of HIF- $2 \alpha$ decreased $\beta$-catenin expression and inhibited hypoxia-induced cell mobility. Moreover, we identified that phosphorylational activation of AKT1 by hypoxia and HIF-2 $\alpha$ was required for Wnt activation upon hypoxia treatment. Downregulation of HIF- $2 \alpha$ and $\beta$-catenin reduced colony formation when cells were exposed to long-term hypoxia treatment. Taken together, our data support that hypoxia activates PI3K/AKT as well as Wnt signaling in a HIF- $2 \alpha$-dependent manner, thus elevating the resistance of lung cancer cells to chronic hypoxia-induced stress.
\end{abstract}

Correspondence to: Dr Cheng-Wen Wu, National Yang-Ming University, No. 155, Sec. 2, Linong Street, Taipei 11221, Taiwan, R.O.C. E-mail: cwwu@ym.edu.tw

Dr Chun-Fu Hong, Department of Long-Term Care, National Quemoy University, No. 1, University Rd., Jinning Township, Kinmen County 89250, Taiwan, R.O.C.

E-mail: cfhong@nqu.edu.tw

Abbreviations: HIF, hypoxia-induced factor; EMT, epithelialmesenchymal transition; VSVG, vesicular stomatitis virus $\mathrm{G}$ protein; PI, propidium iodide; DFO, desferrioxamine

Key words: hypoxia, Wnt signaling, HIF, lung cancer, AKT1

\section{Introduction}

Lung cancer is the leading cause of cancer-related death worldwide. Lung adenocarcinoma is the most common type of lung cancer and is highly associated with smoking. Nonetheless, among non-smokers, $>60 \%$ lung cancers are adenocarcinoma as well (1). With regard to the processes of cancer metastasis and acquired drug resistance, genetic variations and microenvironmental cues are increasingly gaining attention (2). Tumor hypoxia, a prominent microenvironmental cue, has long been associated with increased malignancy, poor prognosis and resistance to chemotherapy $(3,4)$. During tumor growth, tumor cells located away from blood vessels become hypoxic, and recent studies have focused on the mechanisms by which hypoxic tumor cells alter their transcriptional profiles to modulate glycolysis, proliferation, survival, migration and invasion to resist this hypoxic stress.

Hypoxia-inducible factors (HIFs) are $\mathrm{O}_{2}$-regulated transcriptional factors that play critical roles in low-oxygen adaptive mechanism. Increased levels of HIFs have been identified in many solid tumors, such as brain, breast, cervical, gastrointestinal, lung, oropharyngeal, and ovarian cancers $(3,5,6)$. As HIFs induce expression of angiogenic factors and lead to angiogenesis, tumors can acquire more oxygen and nutrients for survival and proliferation $(7,8)$. Two major HIFs, HIF-1 $\alpha$ and HIF-2 $\alpha$, have similar structures and regulate both unique and common target genes. HIF- $1 \alpha$ specifically regulates glycolytic genes, including phosphoglycerate kinase (PGK), lactate dehydrogenase A (LDHA), pyruvate dehydrogenase kinase 1 (PDK1), carbonic hydrase-9 (CA IX) and BNIP3. HIF-2 $\alpha$ exclusively regulates the Pou transcription factor Oct-4, cyclin D1, and transforming growth factor $\alpha$ (TGF- $\alpha$ ) (9-12). Overall, hypoxia increases tumor malignancy and metastasis via activation of multiple hypoxia-responsive genes to regulate cancer cell proliferation, cell survival, and spread.

Tumor hypoxia has been associated with Wnt signaling pathway in the modulation of cancer progression. The Wnt pathway is one of the fundamental mechanisms that regulate cell proliferation, polarity, and fate determination during embryonic development and tissue homeostasis $(13,14)$. In the absence of Wnt ligands, $\beta$-catenin adheres to the plasma membrane and cytoplasmic $\beta$-catenin is kept in a lower level by 
a $\beta$-catenin destruction complex. Upon Wnt ligand stimulation, $\beta$-catenin is stabilized, accumulated, and then translocated to the nucleus to activate its downstream genes (15-17). Loss of Wnt pathway regulation is often linked to birth defects, cancers, and other diseases (18).

Several studies have reported crosstalk between hypoxia and Wnt signaling in the modulation of cancer malignancy and metastasis $(14,18)$. For example, HIF-1 $\alpha$ modulates Wnt/ $\beta$-catenin signaling in hypoxic embryonic stem cells by enhancing $\beta$-catenin activation and expression of the downstream effectors LEF-1 and TCF-1 (19). Moreover, HIF-2 $\alpha$ interacts with $\beta$-catenin and promotes cell proliferation in renal cell carcinoma (RCC) (20). It is worth noting that RCC cells are HIF-1 $\alpha$ deficient and constitutively express HIF- $2 \alpha$ only, whereas hypoxic lung cancer cells express both HIF-1 $\alpha$ and HIF-2 $\alpha$. Although deregulation of Wnt signaling pathway and activation of the hypoxia pathway in lung cancers have been reported, the precise functional crosstalk between hypoxia and Wnt signaling in lung cancer has not yet been determined.

In this study, we investigated the involvement of Wnt signaling pathway and hypoxia in lung cancer progression. Our results showed that Wnt signaling activity is upregulated under hypoxia, and $\beta$-catenin is stabilized and translocated into the nucleus to stimulate expression of downstream Wnt target genes. We further identified that HIF- $2 \alpha$ is the major effector and downregulation of HIF- $2 \alpha$ and $\beta$-catenin reduces cell migration, invasion and colony formation upon hypoxia treatment. Our results also discovered that hypoxia-induced AKT1 phosphorylation is responsible for Wnt signaling activation upon hypoxia treatment. Based on these observations, we suggest that HIF- $2 \alpha$ and $\beta$-catenin cooperatively play essential roles in resisting hypoxia-induced stress in lung cancer cells.

\section{Materials and methods}

Construction of DNA plasmids. A $\beta$-catenin gene (CTNNB1) fragment was PCR-amplified from a human cDNA library using a PCR primer set (forward, 5'-GGATCCATGGCTAC TCAAGCTGATTTGATG-3'; reverse, 5'-GTCGACTCACTT ATCGTCGTCATCCTTGTA-3'), and this fragment was then subcloned into the HR'-puro vector to generate the HR'- $\beta$-catenin-puro plasmid. The plasmids HR'-HIF- $1 \alpha$ P402A/P564A-puro and HR'-HIF-2 $\alpha$-P405A/P531A-puro, which carry constitutively expressed HIF- $1 \alpha$ and HIF- $2 \alpha$, were subcloned from HA-HIF-1 $\alpha$-P402A/P564A-pBabe-puro and HA-HIF-2 $\alpha-\mathrm{P} 405 \mathrm{~A} / \mathrm{P} 531 \mathrm{~A}-\mathrm{pBabe}$-puro (obtained from Addgene), into the HR'-puro vector. Lentiviral plasmids pLKO.1-shHIF-1 $\alpha$ (TRCN0000003810; TRCN0000000819), pLKO.1-shHIF-2 $\alpha$ (TRCN0000003805; TRCN0000003806) and pLKO.1-sh $\beta$-catenin (TRCN0000314990; TRCN 0000314991) were obtained from the RNAi core facility (Academia Sinica, Taipei, Taiwan).

Cell culture. A549, H1975, and HEK293T cell lines were obtained from the American Type Culture Collection (ATCC). Human lung adenocarcinoma cell lines A549 and H1975 were cultured at $37^{\circ} \mathrm{C}$ in $5 \% \mathrm{CO}_{2}$ in RPMI-1640 supplemented with $10 \%$ fetal bovine serum (FBS) and $1 \%$ penicillin/streptomycin. HEK293T cells were maintained at $37^{\circ} \mathrm{C}$ in $5 \% \mathrm{CO}_{2}$ in DMEM supplemented with $10 \% \mathrm{FBS}$ and $1 \%$ penicillin/streptomycin.
Lentiviral preparation and infection. Lentiviral preparation and infection were performed as previously described (21). Briefly, $10 \mu \mathrm{g}$ of the HR'-puro-based or pLKO.1-based lentiviral vector, together with $9 \mu \mathrm{g}$ of $\Delta 8.9$ plasmid and $2.5 \mu \mathrm{g}$ of vesicular stomatitis virus $G$ protein (VSVG) plasmid, was co-transfected into HEK293T cells in a 100-mm culture dish using a standard calcium phosphate transfection protocol. The culture medium was replaced with fresh medium $16 \mathrm{~h}$ after transfection, and the virus-containing supernatants were collected $48 \mathrm{~h}$ after transfection. Lentiviral infection was performed by adding virus solution to the cells in the presence of $8 \mathrm{ng} / \mathrm{ml}$ polybrene. Fresh culture medium containing puromycin $(2.0 \mu \mathrm{g} / \mathrm{ml})$ was added to the cells at $24 \mathrm{~h}$ after infection. The surviving cells were pooled and cultured for further analysis.

$R N A$ isolation and reverse transcription-PCR. Total RNA was extracted from cultured cells using MaestroZol ${ }^{\mathrm{TM}} \mathrm{RNA}$ extraction reagent (OmicsBio, Taipei, Taiwan) according to the manufacturer's protocol. Extracted RNA was then reversetranscribed into cDNA using NCode $^{\mathrm{TM}}$ VILO $^{\mathrm{TM}}$ miRNA cDNA Synthesis kit (Invitrogen) and T3 Thermocycler (Biometra).

Quantitative real-time PCR and primer sequence. qPCR amplification was performed by the $\mathrm{NCode}^{\mathrm{TM}}$ Express SYBR ${ }^{\circledR}$ GreenER $^{\mathrm{TM}}$ qPCR Reagent System (Invitrogen), and Universal Probe Library system (Roche). The following primer sequences are listed below. For UPL system: 18s (Probe: \#48) forward5'-GCA ATT ATT CCC CAT GAA CG-3', reverse-5'-GGG ACT TAA TCA ACG CAA GC-3'; CTNNB1 (Probe: \#21) forward-5'-GCT TTC AGT TGA GCT GAC CA-3', reverse5'-CAA GTC CAA GAT CAG CAG TCT C-3'. For SYBR Green: N-cadherin forward-5'-CCT TGA GCT CCC TTA ATT CC-3', reverse-5'-CCA CCA TAC ATG TCA GCA AG-3'; c-Jun forward-5'-CGG AGA GGA AGC GCA TGA-3', reverse5'-ACC TGT TCC CTG AGC ATG TTG-3'; cyclin D1 (CCND1) forward-5'-CCG AGA AGC TGT GCA TCT ACA C-3', reverse-5'-CGC CTC TGG CAT TTT GGA-3'; GAPDH forward-5'-CGA CCA CTT TGT CAA GCT CA-3'; reverse5'-AGG GGT CTA CAT GGC AAC TG-3'.

Transient transfection and reporter assay. A total of $1 \mu \mathrm{g}$ DNA was added per well of a 24-well culture dish, and transient transfection was performed using the jetPEI ${ }^{\mathrm{TM}}$ transfection reagent (Polyplus-Transfection Inc.) according to the manufacturer's protocol. TOPFlash, a common Wnt activity reporter plasmid expressing firefly luciferase, was co-transfected with plasmids expressing the desired protein into 24-well plates pre-seeded with lung cancer cells. In each well, an SV40-Renilla-luc plasmid expressing Renilla luciferase was co-transfected as a control for transfection efficiency normalization. Luciferase activity assays were performed using a Dual-Luciferase ${ }^{\circledR}$ Reporter Assay System (Promega) according to the manufacturer's protocol. Transfection of each construct and the reporter plasmid was performed in triplicate in each assay. Bars represent the averages of the normalized values, with error bars indicating the range.

Protein extraction and immunoblot. Immunoblot analysis was performed as previously described (21). Briefly, equal amounts 

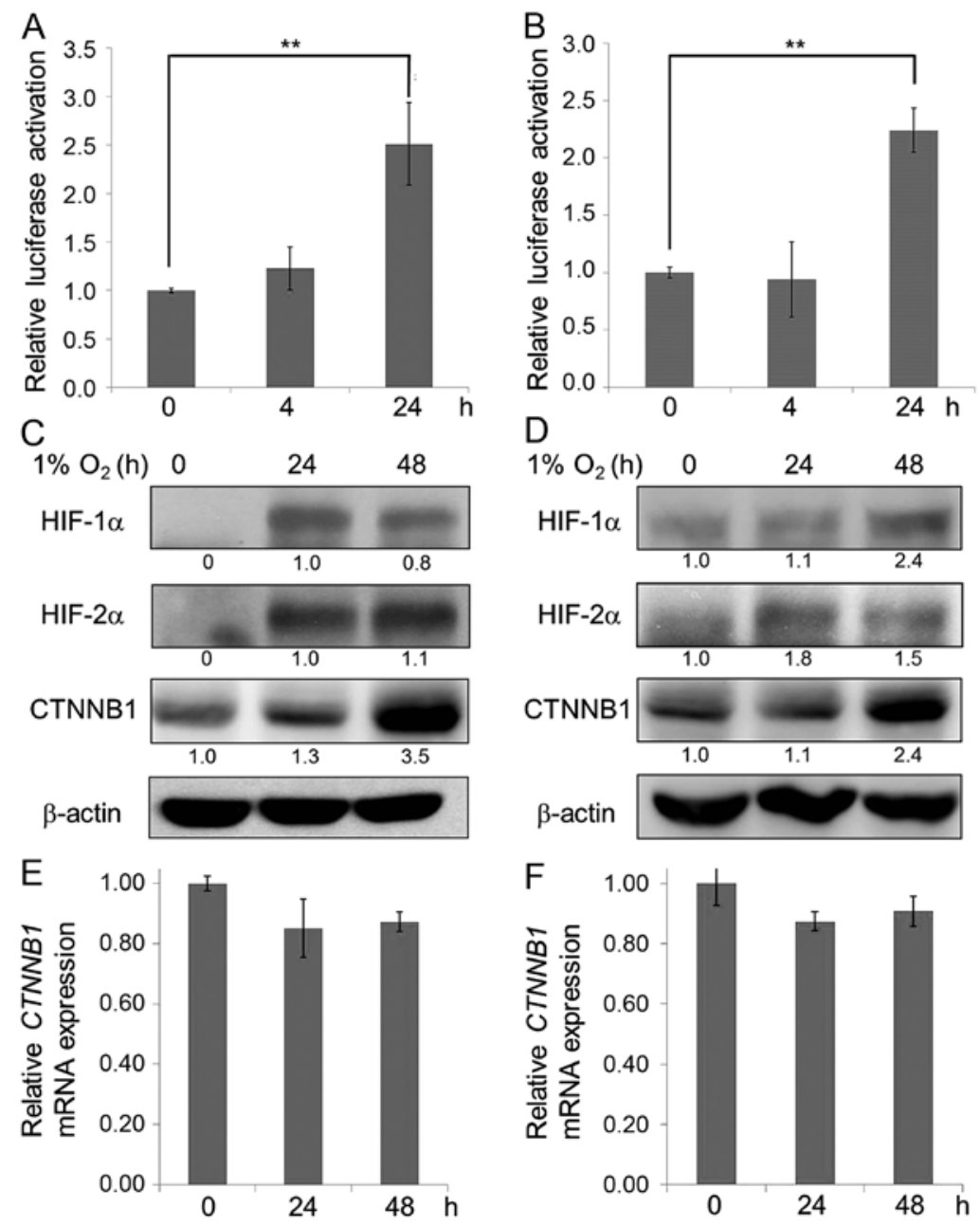

Figure 1. Wnt reporter activity is activated and $\beta$-catenin is upregulated by hypoxia treatment in lung adenocarcinoma cells. (A) TOPFlash (100 ng) and SV40-Renilla-luc $(50 \mathrm{ng}$ ) plasmids were added to 24 -well plates containing pre-seeded A549 cells, and the transfected cells were incubated for the indicated time $(0,4$, and $24 \mathrm{~h})$ in a $1 \% \mathrm{O}_{2}$ hypoxia chamber. Luciferase activity was then determined. (B) TOPFlash reporter activity was also examined in $\mathrm{H} 1975$ cells under hypoxia in an identical experimental designs (A). (C) The protein levels of HIF-1 $\alpha$, HIF-2 $\alpha$ and $\beta$-catenin (CTNNB1) were examined by western blot analysis after the indicated hypoxia treatment time in A549 cells. $\beta$-actin was used as a loading control. (D) The protein levels of HIF-1 $\alpha$, HIF-2 $\alpha$ and $\beta$-catenin (CTNNB1) were examined by western blot analysis after the indicated hypoxia treatment time in $\mathrm{H} 1975$ cells. $\beta$-actin was used as a loading control. (E) CTNNB1 mRNA expression level was not significantly changed in A549 cells upon hypoxia treatment. (F) CTNNB1 mRNA expression level was not significantly changed in $\mathrm{H} 1975$ cells upon hypoxia treatment. Data represent the means \pm SD from three independent experiments. ${ }^{* *} \mathrm{P}<0.01$. In all cases, $1 \%$ $\mathrm{O}_{2}$ was the hypoxic condition. All western blot signal intensities were quantified by ImageJ and further normalized with $\beta$-actin.

of proteins were resolved on an SDS polyacrylamide gel and transferred onto a nitrocellulose membrane. Membranes were then probed with the indicated primary antibodies and appropriate secondary antibodies. The specific signals were visualized by LAS-3000 CCD-imaging system (Fujifilm). The following primary antibodies were used: anti-HIF-1 $\alpha$ (2015-1, Epitomics); anti-HIF-2 $\alpha$ (ab199, Abcam); anti-AKT1 (1081, Epitomics); anti-Phospho-AKT1 Ser473 (2118-1, Epitomics); anti- $\beta$-actin (GTX110564, GeneTex); $\gamma$-tubulin (1878-1, Epitomics); anti- $\beta$-catenin (GTX61089, GeneTex); anti-Lamin A/C (346) (Sc-7293, Santa Cruz Biotechnology). All western blot signal intensities were quantified by NIH Image J software and further normalized with $\beta$-actin (22).

Wound healing cell migration assay. A Culture-Insert (Ibidi, Martinsried, Germany) was placed in a 6-well culture plate, and $3 \times 10^{4}-4 \times 10^{4}$ cells were seeded in each Culture Insert. After $24 \mathrm{~h}$, the Culture-Insert was removed using sterile tweezers, and a $500-\mu \mathrm{m}$ single wound was created in the center of the cell monolayer. After the desired time of incubation, the cells that had migrated into the wound area or protruded from the border of the wound were visualized and photographed under an inverted microscope. The experiment was performed at least three times independently. The area of scratch region was calculated by NIH ImageJ software (23). The relative ratio of scratch area was represented as the ratio of the remaining scratch area at the indicated time points to the whole scratch area at $0 \mathrm{~h}$.

Invasion assay. Matrigel ( $5 \mathrm{mg} / \mathrm{ml})$ (BD Falcon) was diluted in cold 10\% Nuserum (BD Falcon) RPMI and then coated onto a Transwell device ( $8 \mu \mathrm{m}$ pore size, BD Falcon). After incubation at $37^{\circ} \mathrm{C}$ for $30 \mathrm{~min}, 300 \mu 110 \%$ Nuserum RPMI was added into the Transwell. Each Transwell device was then placed into a well containing $700 \mu 110 \%$ Nuserum RPMI in a 24-well plate; $2.5 \times 10^{4}$ cells were seeded into each Transwell and incubated at $37^{\circ} \mathrm{C}$ for $24 \mathrm{~h}$. The cells were then fixed with methanol at $-20^{\circ} \mathrm{C}$ for $20 \mathrm{~min}$ and then stained with propidium 
iodide (PI, $50 \mu \mathrm{g} / \mathrm{ml}$, Sigma-Aldrich). Cells that had penetrated the filter and attached to the other side were counted.

Cell cycle analysis. Briefly, cells infected with desired lentivirus were selected by puromycin. After $48 \mathrm{~h}$, cells were harvested and then fixed with $75 \%$ ethanol overnight at $-4^{\circ} \mathrm{C}$. The fixed cells were incubated with PI staining solution containing RNase A (1 mg/ml, Sigma-Aldrich) and PI $(50 \mu \mathrm{g} / \mathrm{ml})$ for $30 \mathrm{~min}$. Cell cycle status was then examined by flow cytometry on a BD FACSCanto (BD Bioscience) and analyzed by ModFit software.

Statistical analysis. The results were presented as the mean \pm standard deviation of at least 3 independent experiments. Student's t-test was performed to analyze the differences between groups. $\mathrm{P}<0.05$ and $\mathrm{P}<0.01$ were considered to be statistically significant.

\section{Results}

Wnt signaling activity is upregulated by hypoxia. As recent studies indicated that interaction between hypoxia and Wnt signaling promotes cell proliferation in RCC (20), we examined whether the effect of hypoxia $\left(1 \% \mathrm{O}_{2}\right)$ on the Wnt pathway exists in lung adenocarcinoma cells. Reporter assays showed that hypoxia upregulated Wnt reporter (TOPFlash) luciferase activity in both A549 and H1975 lung adenocarcinoma cells (Fig. 1A and B). $\beta$-catenin is the major transcriptional co-activator in the Wnt signaling pathway, and therefore we examined whether hypoxia upregulated expression of $\beta$-catenin. The results showed that hypoxia stabilized HIF-1 $\alpha$ and HIF- $2 \alpha$ and upregulated the protein level of $\beta$-catenin in both A549 and H1975 cells (Fig. 1C and D). We further identified that the increase of $\beta$-catenin expression under hypoxia is due to a post-translational regulation since no significant difference in the mRNA level of $\beta$-catenin under hypoxia compared to normoxia was observed in A549 or H1975 cells (Fig. 1E and F). Together, our data suggest that hypoxia activates Wnt signaling by stabilizing the $\beta$-catenin protein level via a post-translational modification rather than by de novo protein synthesis.

$\beta$-catenin translocates into the nucleus upon hypoxia treatment. Next, an immunofluorescence assay was performed to assess the distribution of $\beta$-catenin upon hypoxia treatment. We detected $\beta$-catenin at the cell membrane and cell-cell junction in normoxic A549 and H1975 cells (Fig. 2A and B, upper panels), whereas $\beta$-catenin was found in the nucleus in hypoxic cells (Fig. 2A and B, low panels). Nuclear translocation of $\beta$-catenin was also detected in the nuclear fraction (Fig. 2C). These data show that upon hypoxia treatment, $\beta$-catenin translocates into the nucleus to stimulate expression of downstream Wnt target genes.

Hypoxia induces Wnt signaling in a HIF-2 $\alpha$ dependent manner. As HIF- $1 \alpha$ and HIF-2 $\alpha$ are two major effectors of hypoxia, we sought to define which factor is more significant in activation of hypoxia-induced Wnt signaling. HIF- $1 \alpha$, HIF- $2 \alpha$ and $\beta$-catenin, together with a Wnt reporter plasmid TOPFlash, were transiently co-overexpressed in A549 cells, and luciferase activity was then
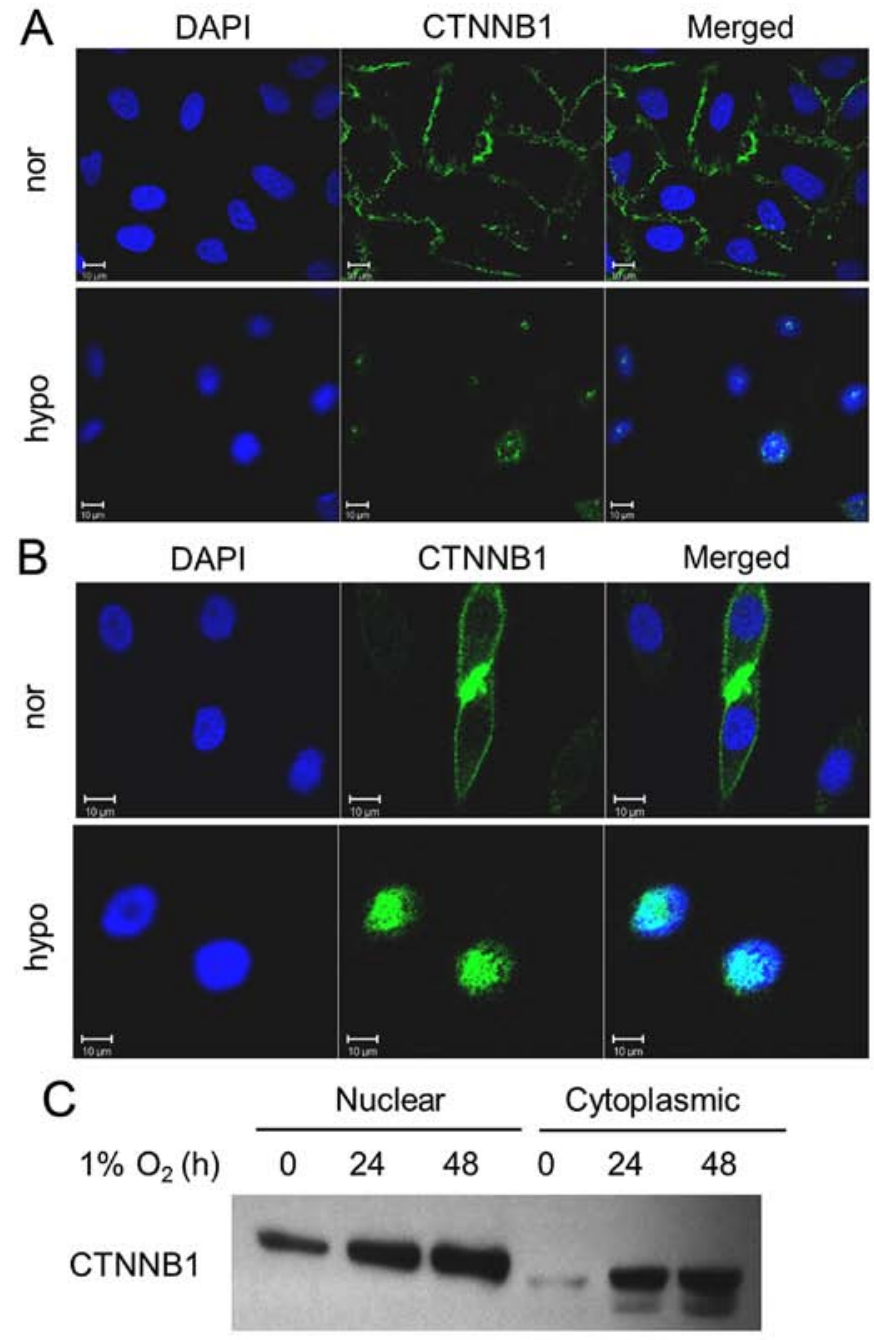

Lamin A/C

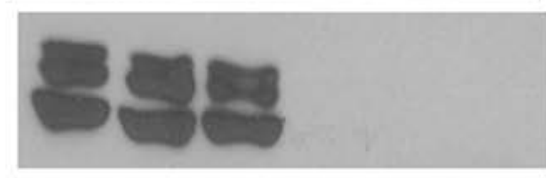

$\gamma$-tubulin

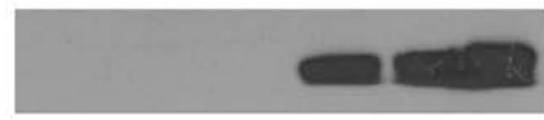

Figure 2. $\beta$-catenin was accumulated and translocated to the nucleus upon hypoxia treatment. (A) Immunofluorescence staining for normoxic (nor) and hypoxic (hypo) A549 cells (exposing to $1 \% \mathrm{O}_{2}$ for $48 \mathrm{~h}$ ) was performed using an anti- $\beta$-catenin antibody (green). All images were examined by confocal microscopy. (B) Immunofluorescence staining for normoxic (nor) and hypoxic (hypo) H1975 cells was performed. (C) Nuclear and cytoplasmic fractions from hypoxic A549 cells were used to examine $\beta$-catenin expression by western blot analysis. Lamin A/C and $\gamma$-tubulin were used as loading controls. The nucleus was labeled with DAPI (blue). Scale bar, $10 \mu \mathrm{m}$. In all cases, $1 \% \mathrm{O}_{2}$ was the hypoxic condition.

determined. Our results showed that overexpression of HIF-2 $\alpha$ and $\beta$-catenin each activated $W n$ reporter activity and that co-overexpression of HIF- $2 \alpha$ and $\beta$-catenin further enhanced this activity (Fig. 3A). Western blot analysis also showed significant upregulation of the expression level of $\beta$-catenin in HIF-2 $\alpha$-overexpressing A549 cells (Fig. 3B). Real-time qPCR analysis showed that the mRNA level of Wnt downstream genes, such as c-Jun and $\mathrm{N}$-cadherin, were significantly enhanced in HIF-2 $\alpha$-overexpressing A549 cells (Fig. 3C). In addition, ectopic overexpression of HIF- $2 \alpha$ and $\beta$-catenin using 

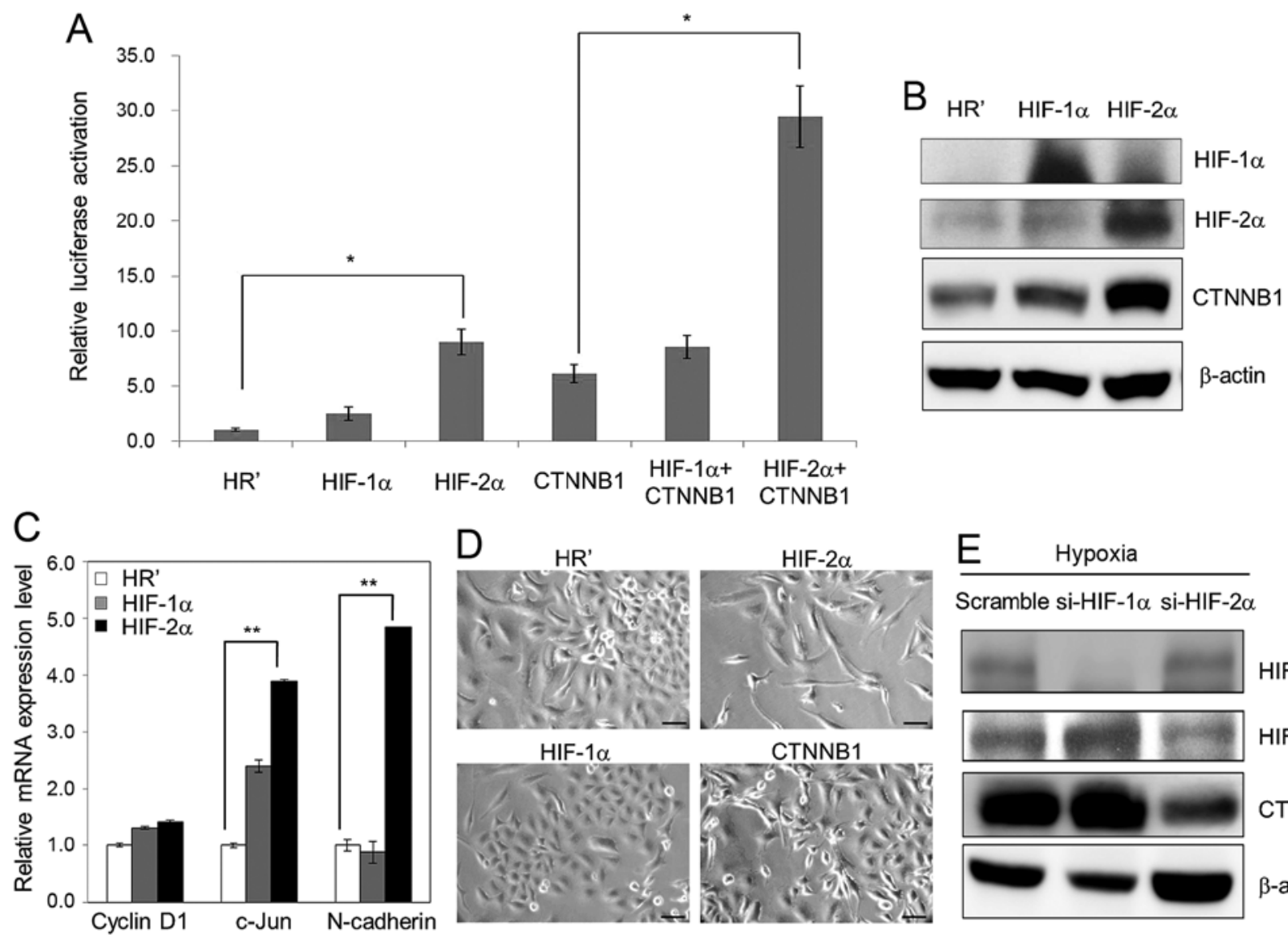
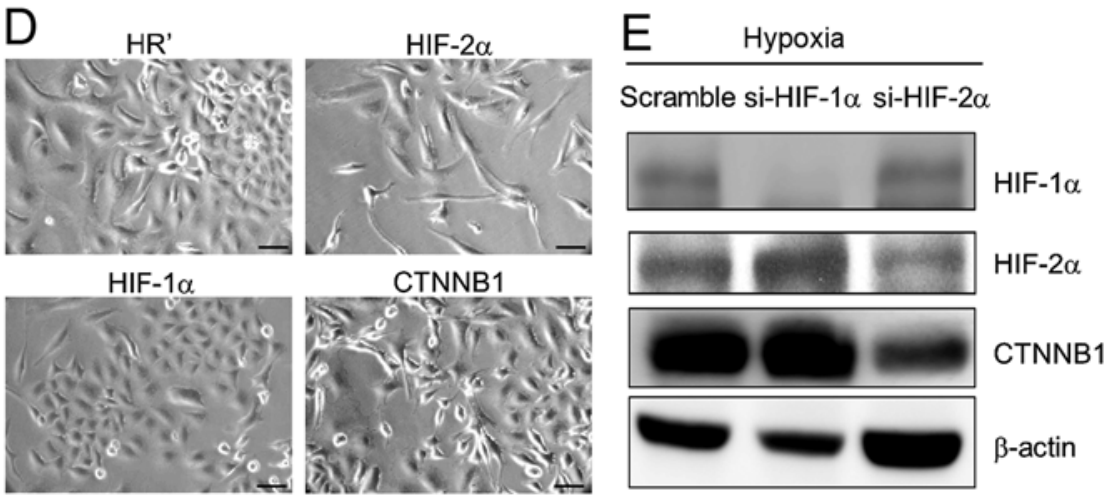

Figure 3. HIF-2 $\alpha$ is the major effector of Wnt signaling activation upon hypoxia treatment. (A) TOPFlash (100 ng) and SV40-Renilla-luc (50 ng) plasmids were transiently co-transfected with plasmids carrying HIF-1 $\alpha, \mathrm{HIF}-2 \alpha$ and $\beta$-catenin (CTNNB1) into A549 cells. Reporter luciferase activity assay was examined at $48 \mathrm{~h}$ after transfection. (B) Lentiviral-based ectopic expression of HR' control, HIF-1 $\alpha$ and HIF-2 $\alpha$ was performed using A549 cells. Protein expression of HIF-1 $\alpha$, HIF- $2 \alpha$ and $\beta$-catenin was then examined by western blotting. (C) The mRNA level of cyclin D1, c-Jun and N-cadherin was examined in HR' control-, HIF-1 $\alpha$ - and HIF-2 $\alpha$-overexpressing A549 cells. (D) Lentiviral-based ectopic expression of HR' control, HIF-1 $\alpha$, HIF-2 $\alpha$ and $\beta$-catenin was performed in A549 cells. The morphology of all stable cell lines was examined by microscopy. Scale bar, $50 \mu$ m. (E) Lentiviral-based knockdown of scramble control, si-HIF- $1 \alpha$ and si-HIF-2 $\alpha$ was performed using A549 cells, and the protein expression of HIF-1 $\alpha$, HIF- $2 \alpha$ and $\beta$-catenin under hypoxia was then examined by western blot analysis. In all cases, $1 \% \mathrm{O}_{2}$ was the hypoxic condition. $\beta$-actin was used as a loading control. Data represent as the means $\pm \mathrm{SD}$ from three independent experiments. ${ }^{*} \mathrm{P}<0.05$. $^{* *} \mathrm{P}<0.01$.

a lentiviral system induced morphological changes in A549 cells, whereas overexpression of HR' control and HIF-1 $\alpha$ had no significant effect (Fig. 3D). After performing lentiviral-based RNA silencing of HIF- $1 \alpha$ and HIF- $2 \alpha$ in A549 cells, upregulation of $\beta$-catenin upon hypoxia $\left(1 \% \mathrm{O}_{2}\right)$ was inhibited in the cells knocked down for HIF-2 $\alpha$ but not in those knocked down for HIF-1 $\alpha$ (Fig. 3E). These results indicate that HIF-2 $\alpha$ might play an even more essential role in hypoxia-induced morphological changes and Wnt activation in lung cancer cells.

$H I F-2 \alpha$ and $\beta$-catenin are essential for hypoxia-induced cell migration and invasion. Next we attempted to define whether Wnt signaling, as well as HIF-1 $\alpha$ and HIF- $2 \alpha$, has an important function in hypoxia-induced migration of lung cancer cells by knocking down HIF- $1 \alpha$, HIF- $2 \alpha$ and $\beta$-catenin in A549 cells using lentiviral-based small interfering RNAs. Our data showed that knockdown of HIF-1 $\alpha$ did not affect hypoxiainduced cell migration into the scratched area compared to scramble control cells (Fig. 4A and B). In contrast, knockdown of HIF- $2 \alpha$ and $\beta$-catenin both significantly disrupted hypoxiainduced cell migration into the scratched area (Fig. 4C and D). Invasion assays revealed that cells with HIF-1 $\alpha$ knockdown exhibited similar invasion ability compared to scramble control cells upon hypoxia. However, knockdown of HIF-2 $\alpha$ and $\beta$-catenin significantly suppressed hypoxia-induced invasion (Fig. 4E). Stable A549 cell lines carrying HR' control-, HIF- $1 \alpha-$, HIF- $2 \alpha-$, and $\beta$-catenin-overexpression were used to examine their cell migration ability. We did not observe significant differences in migration ability between these cells under normal culture condition, but under low-serum condition $(0.5 \%$ FBS $)$, we observed that overexpression of HIF- $2 \alpha$ and $\beta$-catenin increased cell migration compared to HR' control- and HIF-1 $\alpha$-overexpressing cells (Fig. 4F). These results indicate that Wnt signaling may regulate hypoxiainduced cell migration and invasion via $\beta$-catenin activation in a HIF-2 $\alpha$-dependent manner.

Hypoxia increases $\beta$-catenin expression through the PI3K/ $A K T$ pathway. Previous studies suggested that hypoxia induces the PI3K/AKT pathway in many cell types (24-26). We further examined the effect of hypoxia on the PI3K/AKT pathway in lung cancer cells. Upon hypoxia, phosphorylation of AKT1 (Ser473) was upregulated in A549 cells (Fig. 5A). Furthermore, phospho-AKT1 expression was increased in 

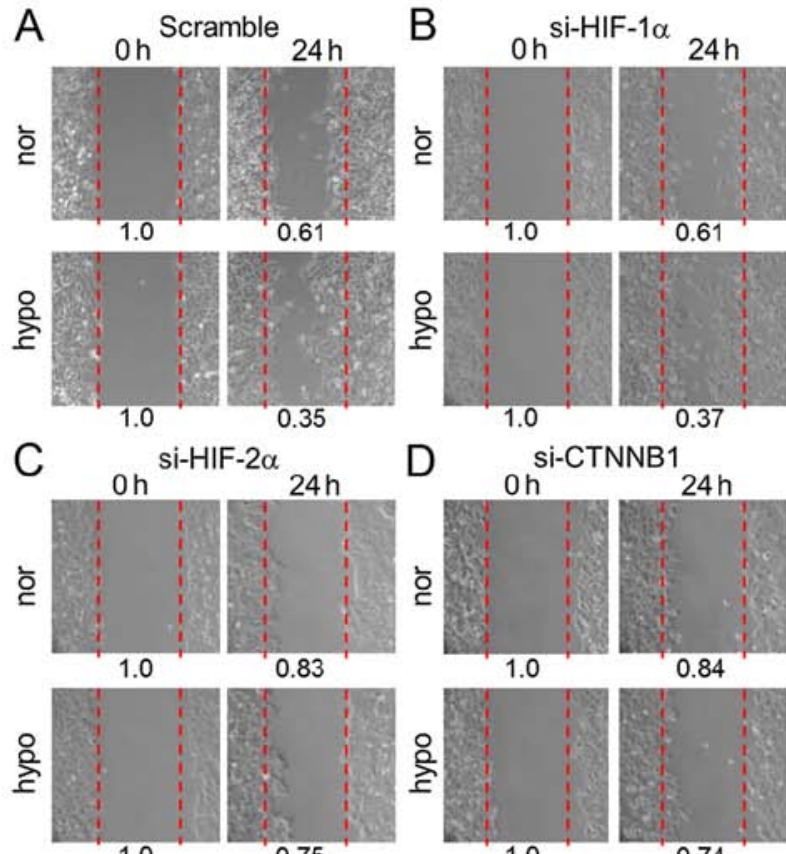

0.84
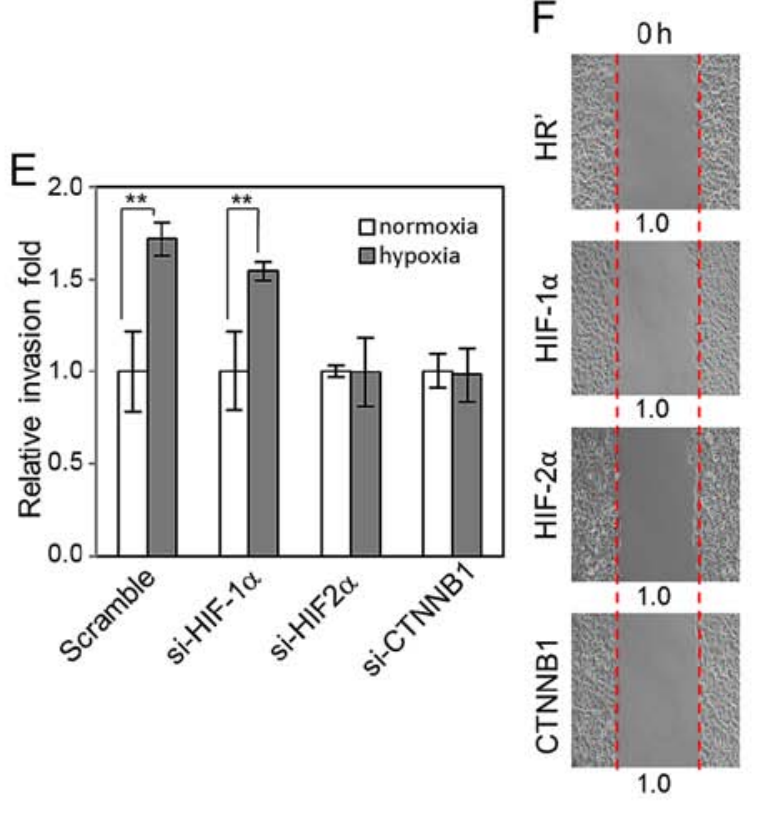

1.0

0.74

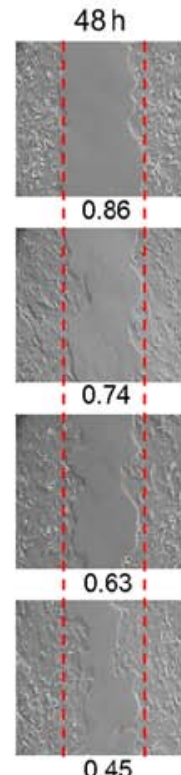

Figure 4. Knockdown of HIF-2 $\alpha$ and $\beta$-catenin disrupts the enhancement of migration and invasion induced by hypoxia. For the wound healing migration assay, lentivirus-infected A549 cells carrying scramble control (A), si-HIF-1 $\alpha$ (B), si-HIF-2 $\alpha$ (C) and si- $\beta$-catenin (si-CTNNB1) (D) were seeded in each well of a Culture-Insert in a 6-well plate. All images were examined by microscopy after $24 \mathrm{~h}$ under normoxia (nor) and hypoxia (hypo). (E) Lentivirus-infected stable cells were seeded in Matrigel-coated Transwell insert. After incubation for $24 \mathrm{~h}$ under normoxia and hypoxia, the Transwell inserts containing invasive cells were fixed with methanol and stained using PI. Cells that migrated through the filter to the other side were counted. Data represent the means \pm SD from three independent experiments. ${ }^{* *} \mathrm{P}<0.01$. (F) HR' control-, HIF-1 $\alpha$-, HIF-2 $\alpha$ - and $\beta$-catenin-overexpressing A549 cells were seeded in a Culture-Insert in RPMI medium containing $0.5 \%$ FBS. All samples were examined by microscopy after $48 \mathrm{~h}$. In all cases, $1 \% \mathrm{O}_{2}$ was the hypoxic condition. Dotted lines indicate the wound borders at the beginning of the assays. The relative ratio of the remaining scratch area to the whole scratch area was calculated and displayed in each diagram.

HIF-2 $\alpha$-overexpressing cells compared to HR' control and HIF-1 $\alpha$-overexpressing cells (Fig. 5B). To define whether the $\mathrm{PI} 3 \mathrm{~K} / \mathrm{AKT}$ pathway is involved in hypoxia-induced $\beta$-catenin upregulation, a PI3K inhibitor, LY294002 (Sigma-Aldrich), was employed to inhibit AKT1 phosphorylation. The results showed decreased expression of $\beta$-catenin and AKT1 phosphorylation upon LY294002 treatment, even under hypoxia (Fig. 5C). Our results suggest that PI3K/AKT pathway is activated by HIF- $2 \alpha$ and is essential in hypoxia-induced Wnt signaling activation.

$H I F-2 \alpha$ and $\beta$-catenin protect lung cancer cells from long-term hypoxia-induced stress. To further investigate the significant role of HIF- $2 \alpha$ and $\beta$-catenin under hypoxia, we incubated cells with desferrioxamine (DFO, Sigma-Aldrich) to mimic long-term exposure to hypoxia. When incubated in $100 \mu \mathrm{M}$ DFO for 24 and 48 h, HIF- $1 \alpha$ and HIF-2 $\alpha$ were successfully activated in A549 cells (Fig. 6A, left panel). Our results showed that after 21 days of incubation with $100 \mu \mathrm{M}$ DFO in culture, knockdown of HIF- $2 \alpha$ and $\beta$-catenin in A549 cells significantly reduced their colony numbers compared to scramble control and HIF-1 $\alpha$-silenced cells in colony formation assays (Fig. 6A, right panel); additionally, upon chronic hypoxic stress, HIF- $2 \alpha$ - and $\beta$-catenin-overexpressing A549 cells possessed higher colony numbers compared to HR' control cells, and co-overexpression of HIF- $2 \alpha / \beta$-catenin can enhance the growth advantage even higher than overexpression of HIF-1 $\alpha$ or $\beta$-catenin alone (Fig. 6B). As our results suggested that $\beta$-catenin plays an essential role in controlling cell growth under hypoxic stress, we further examined the effect of $\beta$-catenin in lung cancer cell growth by examining cell cycle progression in $\beta$-catenin-silenced A549 and H1975 cells. The results showed increase in the sub-G1 population in $\beta$-cateninsilenced cells, indicating that $\beta$-catenin has an essential role in aiding the survival of lung cancer cells (Fig. 6C). Together, these data suggest that HIF- $2 \alpha$ and $\beta$-catenin are essential factors in resisting chronic hypoxia-induced cell death and crosstalk between hypoxia and Wnt pathway is involved in lung cancer progression.

\section{Discussion}

Intratumoral hypoxia has long been associated with increased malignancy and cancer metastasis. Both HIF- $1 \alpha$ and HIF-2 $\alpha$ are overexpressed in many cancer types and are associated with poor prognosis in cancers of the breast, brain, cervix, ovary, and uterus (27-30). Crosstalk between hypoxia and Wnt signaling has also been noted. However, most studies focus on either HIF- $1 \alpha$ or HIF- $2 \alpha$, respectively. In this study, we determined that HIF-2 $\alpha$ is the major effector of lung cancer cells in Wnt signaling activation under hypoxia and crosstalk between Wnt pathway and hypoxia participates in lung cancer progression processes, such as metastasis and cell survival. Overexpression of HIF-2 $\alpha$ in lung cancer cells increases $\beta$-catenin expression and induces morphological changes similar to EMT. Knockdown of HIF-2 $\alpha$ decreases $\beta$-catenin expression and inhibits hypoxia-induced cell migration and invasion, whereas no significant effect was observed with 


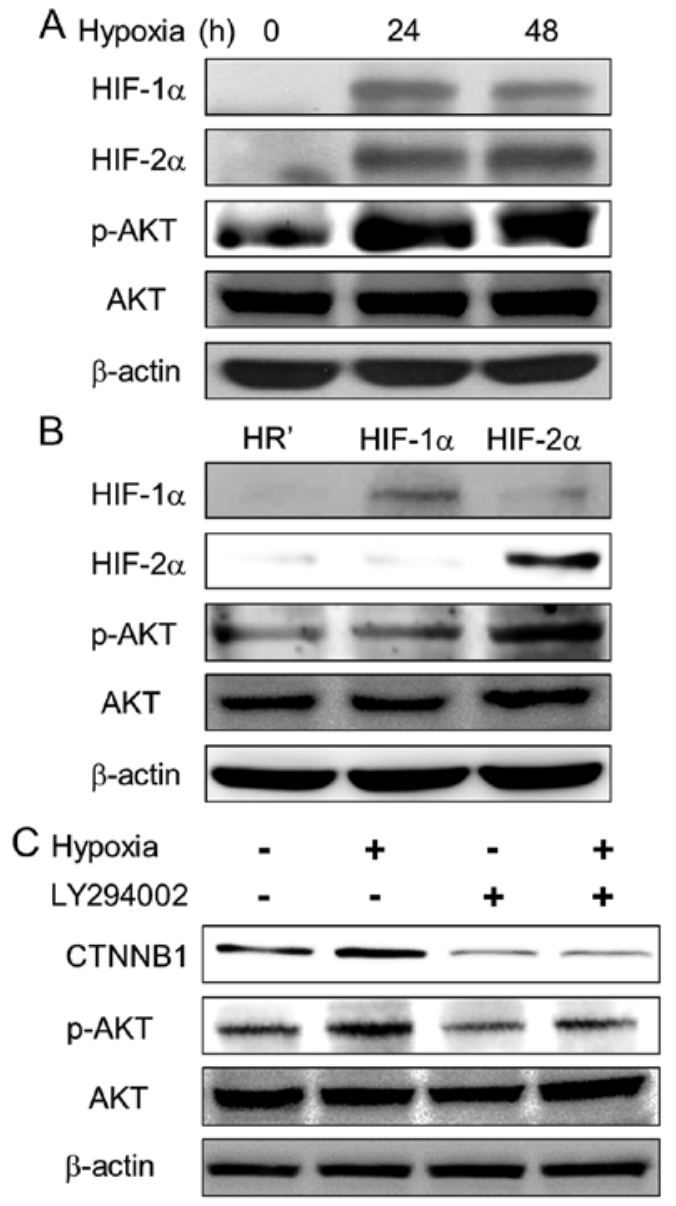

Figure 5. The PI3K/AKT pathway is involved in hypoxia-induced $\beta$-catenin protein upregulation. (A) A549 cells were treated with hypoxia for 24 or $48 \mathrm{~h}$. The expression levels of HIF-1 $\alpha$, HIF- $2 \alpha$ and phospho-AKT S473 (p-AKT) were then examined by western blot analysis. (B) A549 cell lines overexpressing HR' control, HIF-1 $\alpha$ and HIF- $2 \alpha$ were cultured in RPMI medium for $48 \mathrm{~h}$ and lysed; the expression levels of HIF- $1 \alpha$, HIF-2 $\alpha$ and p-AKT were then examined. (C) The PI3K inhibitor LY294002 was applied to inhibit endogenous AKT activity. The expression levels of $\beta$-catenin (CTNNB1), $\mathrm{p}$-AKT were then examined by western blot analysis. $\beta$-actin was used as a loading control.

knockdown of HIF-1 $\alpha$ (Fig. 4A-E). HIF-1 $\alpha$ is only responsible for a minor effect of hypoxia-induced cell invasion (Fig. 4E), and therefore it is reasonible that si-HIF- $2 \alpha$ can abolish the majority of hypoxia-induced cell invasion. Our results also showed that si-CTNNB1 abolished hypoxia-induced invasion. It is not surprising since $\beta$-catenin is an important factor in WNT signaling pathway as well as, in this study, hypoxia pathway. We also proved that hypoxia increases $\beta$-catenin expression, resulting in $\beta$-catenin translocation to the nucleus to stimulate Wnt downstream target genes. Thus, we demonstrate that hypoxia increases Wnt signaling activity and tumor cell malignancy by stabilizing $\beta$-catenin protein levels in a HIF-2 $\alpha$-dependent manner.

The roles of hypoxia in regulating hypoxic cell death or promoting cell survival remain controversial. For example, hypoxia-induced cell death has been observed in E1 A and Ha-ras transformed cell lines, several nonglioma tumor cells, glioblastoma cells, and nontransformed cells (31-33); in addition, HIF-1 $\alpha$ not only activates genes involving in metabolic adaption to hypoxic environment to promote cell survival,
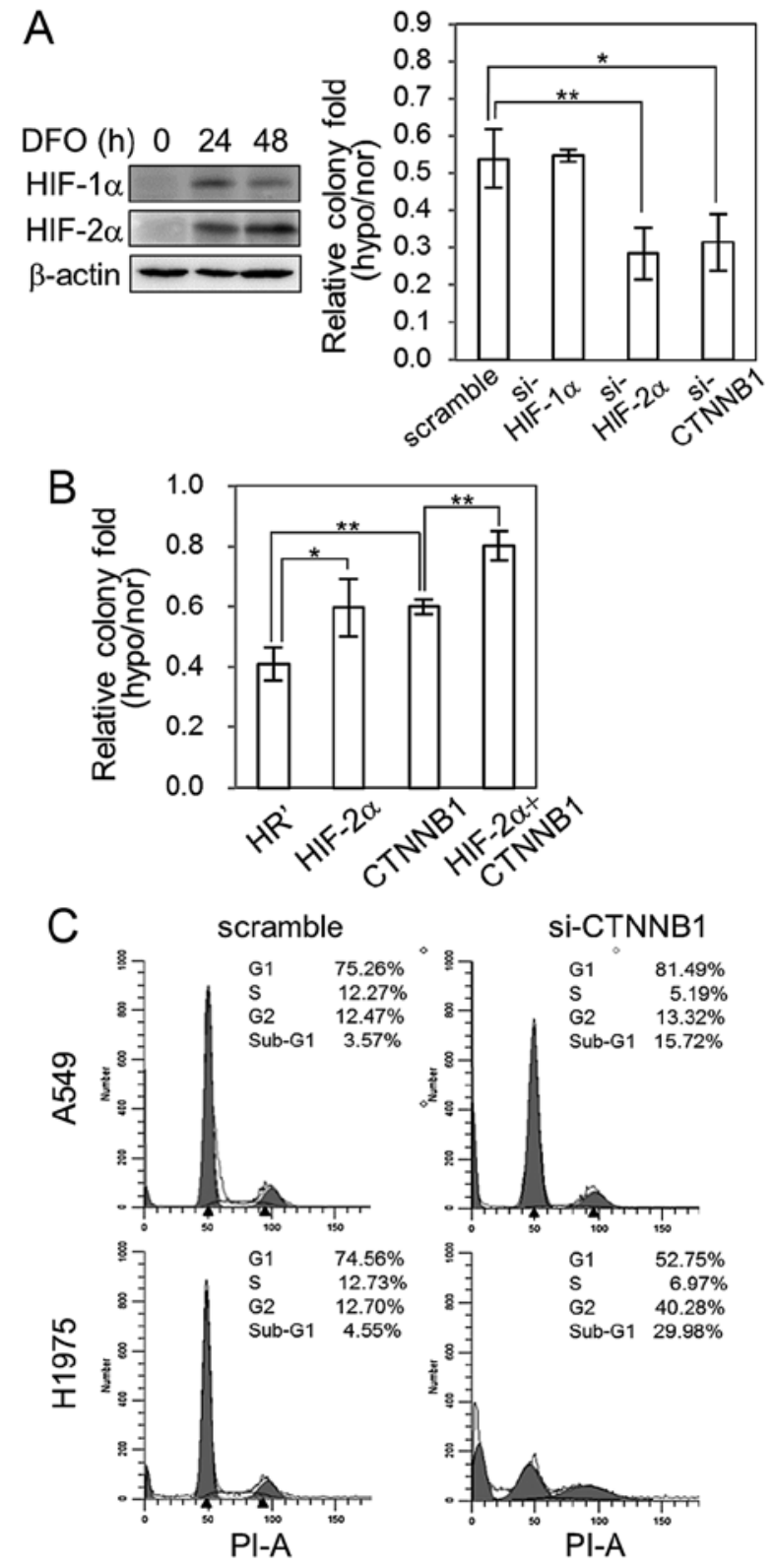

Figure 6. HIF-2 $\alpha$ and $\beta$-catenin are essential in resisting chronic hypoxic stress. (A) Left panel: A549 cells were exposed to $100 \mu \mathrm{M}$ DFO for 24 and $48 \mathrm{~h}$. The expression level of HIF-1 $\alpha$ and HIF- $2 \alpha$ were examined by western blot analysis. $\beta$-actin was used as a loading control. Right panel: A549 cell lines carrying scramble control, si-HIF- $1 \alpha$, si-HIF- $2 \alpha$ and si- $\beta$-catenin (si-CTNNB1) were assessed for colony formation in the presence of $100 \mu \mathrm{M}$ DFO for 21 days. The results are displayed as the ratio of colony numbers between DFO treatment (hypo) and normoxia (nor) for each cell line, respectively. (B) A549 cell lines overexpressing either HR' control. HIF- $2 \alpha, \beta$-catenin or HIF- $2 \alpha / \beta$-catenin were assessed for colony formation in the presence of $100 \mu \mathrm{M}$ DFO for 21 days. The results are displayed as the relative fold of colony numbers between DFO treatment (hypo) and normoxia (nor) for these cell lines, respectively. (C) A549 and H1975 cell lines carrying scramble control and si-CTNNB1 were subjected to cell cycle analysis. Data represent as the means \pm SD from three independent experiments. ${ }^{*} \mathrm{P}<0.05$. ${ }^{* *} \mathrm{P}<0.01$.

but also induces several pro-apoptotic factors, including P53, BNIP3, and BNIP3L, to promote cell death (34). In another perspective, hypoxia-induced autophagy has been reported to play protective roles in cell survival under hypoxic stress (35). The present study provides an alternative survival way in showing that ectopic expression of HIF-2 $\alpha$ upregulates 
AKT1 phosphorylation and inhibition of PI3K/AKT activity by LY294002 reduced hypoxia-induced $\beta$-catenin expression, indicating that PI3K/AKT upregulation is essential for hypoxia-induced Wnt activation. These data suggest that hypoxia-induced $\beta$-catenin upregulation may be important for resistance to hypoxic stress. In colony formation assays using DFO to mimic long-term hypoxia exposure, knockdown of HIF- $2 \alpha$ and $\beta$-catenin significantly reduces colony numbers, whereas overexpression of either HIF- $2 \alpha, \beta$-catenin or HIF- $2 \alpha / \beta$-catenin increases colony numbers. These data further emphasize the significant role of Wnt signaling in resisting hypoxic stress.

In this study, we also examined the expression level of Wnt target genes under HIF-1 $\alpha$ - and HIF-2 $\alpha$-overexpression. While our results showed that overexpression of HIF- $2 \alpha$ upregulates Wnt target genes to a greater extent, it is rather remarkable that not all of Wnt target genes examined were upregulated accordingly, i.e., for example, we were unable to observe a significant elevation of cyclin D1 expression in HIF- $1 \alpha$ - and HIF- $2 \alpha$-overexpressing cells. Cyclin D1 associates with CDK4 to form a protein kinase complex that phosphorylates and inactivates retinoblastoma protein $\mathrm{pRb}$, playing a critical role in regulating cell cycle progression (36). Cyclin D1 is frequently amplified and overexpressed in cancer cells, implying its important role in malignant development (37). The lack of elevated cyclin D1 expression can partially explain why we did not observe a significant growth advantage in HIF- $2 \alpha$ - and $\beta$-catenin-overexpressing cells under normal culture condition. However, when cells were cultured in a high stringent condition such as chronic hypoxia or low serum, overexpression of HIF- $2 \alpha$ and $\beta$-catenin showed elevated migration and colony formation ability.

In summary, our results discriminate the functional differences between HIF- $1 \alpha$ and HIF- $2 \alpha$ in the hypoxic environment and also suggest the involvement of HIF-2 $\alpha$ and the PI3K/AKT pathway in activating Wnt signaling under hypoxia, thus promoting the survival and malignancy of lung cancer cells.

\section{Acknowledgements}

This work was supported by the Ministry of Science and Technology Grant MOST 105-2325-B-010-003, Ministry of Health and Welfare Grant MOHW106-TDU-B-211-144-003, and National Yang Ming University Grant 106AC-P902, Taiwan.

\section{References}

1. Herbst RS, Heymach JV and Lippman SM: Lung cancer. N Eng J Med 359: 1367-1380, 2008.

2. Rohwer $\mathrm{N}$ and Cramer T: Hypoxia-mediated drug resistance: Novel insights on the functional interaction of HIFs and cell death pathways. Drug Resist Updat 14: 191-201, 2011.

3. Semenza GL: Targeting HIF-1 for cancer therapy. Nat Rev Cancer 3: 721-732, 2003.

4. Bertout JA, Patel SA and Simon MC: The impact of $\mathrm{O}_{2}$ availability on human cancer. Nat Rev Cancer 8: 967-975, 2008.

5. Semenza GL: Evaluation of HIF-1 inhibitors as anticancer agents. Drug Discov Today 12: 853-859, 2007.

6. Semenza GL: Oxygen homeostasis. Wiley Interdiscip Rev Syst Biol Med 2: 336-361, 2010

7. Jain RK: Normalization of tumor vasculature: An emerging concept in antiangiogenic therapy. Science 307: 58-62, 2005.
8. Du R, Lu KV, Petritsch C, Liu P, Ganss R, Passegué E, Song H, Vandenberg S, Johnson RS, Werb Z, et al: HIF1a induces the recruitment of bone marrow-derived vascular modulatory cells to regulate tumor angiogenesis and invasion. Cancer Cell 13: 206-220, 2008.

9. Hu CJ, Wang LY, Chodosh LA, Keith B and Simon MC Differential roles of hypoxia-inducible factor $1 \alpha(\mathrm{HIF}-1 \alpha)$ and HIF-2 $\alpha$ in hypoxic gene regulation. Mol Cell Biol 23: 9361-9374, 2003.

10. Warnecke C, Zaborowska Z, Kurreck J, Erdmann VA, Frei U, Wiesener $\mathrm{M}$ and Eckardt KU: Differentiating the functional role of hypoxia-inducible factor (HIF)- $1 \alpha$ and HIF-2 $\alpha$ (EPAS-1) by the use of RNA interference: Erythropoietin is a HIF-2 $\alpha$ target gene in Hep3B and Kelly cells. FASEB J 18: 1462-1464, 2004.

11. Rankin EB, Higgins DF, Walisser JA, Johnson RS, Bradfield CA and Haase $\mathrm{VH}$ : Inactivation of the arylhydrocarbon receptor nuclear translocator (Arnt) suppresses von Hippel-Lindau disease-associated vascular tumors in mice. Mol Cell Biol 25: 3163-3172, 2005.

12. Hu CJ, Iyer S, Sataur A, Covello KL, Chodosh LA and Simon MC: Differential regulation of the transcriptional activities of hypoxia-inducible factor 1 alpha (HIF-1alpha) and HIF-2alpha in stem cells. Mol Cell Biol 26: 3514-3526, 2006.

13. Logan CY and Nusse R: The Wnt signaling pathway in development and disease. Annu Rev Cell Dev Biol 20: 781-810, 2004

14. MacDonald BT, Tamai K and He X: Wnt//-catenin signaling: Components, mechanisms, and diseases. Dev Cell 17: 9-26, 2009.

15. Hecht A, Vleminckx K, Stemmler MP, van Roy F and Kemler R: The p300/CBP acetyltransferases function as transcriptional coactivators of beta-catenin in vertebrates. EMBO J 19: 1839-1850, 2000

16. Takemaru KI and Moon RT: The transcriptional coactivator CBP interacts with beta-catenin to activate gene expression. J Cell Biol 149: 249-254, 2000.

17. Barker N, Hurlstone A, Musisi H, Miles A, Bienz M and Clevers $\mathrm{H}$ : The chromatin remodelling factor Brg-1 interacts with $\beta$-catenin to promote target gene activation. EMBO J 20: 4935-4943, 2001

18. Clevers H: Wnt $/ \beta$-catenin signaling in development and disease. Cell 127: 469-480, 2006.

19. Mazumdar J, O'Brien WT, Johnson RS, LaManna JC, Chavez JC, Klein PS and Simon MC: $\mathrm{O}_{2}$ regulates stem cells through Wnt/ß-catenin signalling. Nat Cell Biol 12: 1007-1013, 2010.

20. Choi H, Chun YS, Kim TY and Park JW: HIF-2 $\alpha$ enhances $\beta$-catenin/TCF-driven transcription by interacting with $\beta$-catenin. Cancer Res 70: 10101-10111, 2010.

21. Hong C-F, Lin S-Y, Chou Y-T and Wu C-W: MicroRNA-7 compromises $\mathrm{p} 53$ protein-dependent apoptosis by controlling the expression of the chromatin remodeling factor SMARCD1. J Biol Chem 291: 1877-1889, 2016.

22. Li S, Yang B, Teguh D, Zhou L, Xu J and Rong L: Amyloid $\beta$ peptide enhances RANKL-induced osteoclast activation through $\mathrm{NF}-\kappa \mathrm{B}, \mathrm{ERK}$, and calcium oscillation signaling. Int J Mol Sci 17: $1683,2016$.

23. Cormier N, Yeo A, Fiorentino E and Paxson J: Optimization of the wound scratch assay to detect changes in murine mesenchymal stromal cell migration after damage by soluble cigarette smoke extract. J Vis Exp 106: e53414, 2015.

24. Alvarez-Tejado M, Naranjo-Suarez S, Jiménez C, Carrera AC, Landázuri MO and del Peso L: Hypoxia induces the activation of the phosphatidylinositol 3-kinase/Akt cell survival pathway in PC12 cells: Protective role in apoptosis. J Biol Chem 276: 22368-22374, 2001.

25. Deguchi JO, Yamazaki H, Aikawa E and Aikawa M: Chronic hypoxia activates the Akt and $\beta$-catenin pathways in human macrophages. Arterioscler Thromb Vasc Biol 29: 1664-1670, 2009.

26. Beitner-Johnson D, Rust RT, Hsieh TC and Millhorn DE: Hypoxia activates Akt and induces phosphorylation of GSK-3 in PC12 cells. Cell Signal 13: 23-27, 2001.

27. Zhong H, De Marzo AM, Laughner E, Lim M, Hilton DA, Zagzag D, Buechler P, Isaacs WB, Semenza GL and Simons JW: Overexpression of hypoxia-inducible factor 1alpha in common human cancers and their metastases. Cancer Res 59: 5830-5835, 1999.

28. Lu X and Kang Y: Hypoxia and hypoxia-inducible factors: Master regulators of metastasis. Clin Cancer Res 16: 5928-5935, 2010.

29. Yang MH, Wu MZ, Chiou SH, Chen PM, Chang SY, Liu CJ, Teng SC and Wu KJ: Direct regulation of TWIST by HIF-1 $\alpha$ promotes metastasis. Nat Cell Biol 10: 295-305, 2008. 
30. Ouyang G,Liu M,Ruan K, Song G, Mao Y and Bao S: Upregulated expression of periostin by hypoxia in non-small-cell lung cancer cells promotes cell survival via the Akt/PKB pathway. Cancer Lett 281: 213-219, 2009

31. Graeber TG, Osmanian C, Jacks T, Housman DE, Koch CJ, Lowe SW and Giaccia AJ: Hypoxia-mediated selection of cells with diminished apoptotic potential in solid tumours. Nature 379: 88-91, 1996.

32. Araya R, Uehara T and Nomura Y: Hypoxia induces apoptosis in human neuroblastoma SK-N-MC cells by caspase activation accompanying cytochrome $\mathrm{c}$ release from mitochondria. FEBS Lett 439: 168-172, 1998.

33. Steinbach JP, Wolburg H, Klumpp A, Probst $\mathrm{H}$ and Weller M: Hypoxia-induced cell death in human malignant glioma cells: Energy deprivation promotes decoupling of mitochondrial cytochrome c release from caspase processing and necrotic cell death. Cell Death Differ 10: 823-832, 2003.
34. Pouysségur J, Dayan F and Mazure NM: Hypoxia signalling in cancer and approaches to enforce tumour regression. Nature 441: 437-443, 2006.

35. Zhang N, Ji N, Jiang W-M, Li ZY, Wang M, Wen JM, Li Y, Chen $X$ and Chen JM: Hypoxia-induced autophagy promotes human prostate stromal cells survival and ER-stress. Biochem Biophys Res Commun 464: 1107-1112, 2015.

36. Musgrove EA, Lee CS, Buckley MF and Sutherland RL: Cyclin D1 induction in breast cancer cells shortens G1 and is sufficient for cells arrested in G1 to complete the cell cycle. Proc Natl Acad Sci USA 91: 8022-8026, 1994.

37. Santarius T, Shipley J, Brewer D, Stratton MR and Cooper CS: A census of amplified and overexpressed human cancer genes. Nat Rev Cancer 10: 59-64, 2010. 